ISSN 1600-5368

\section{Crystal structure of bis\{2-[bis(2-hydroxy- ethyl)amino] ethanol- $\kappa^{3} \mathrm{O}, \mathrm{N}, \mathrm{O}^{\prime}$ \}zinc terephthalate}

\author{
Ya-Ping Li, ${ }^{a}$ Hu Zang, ${ }^{b}$ Dajun Sun, ${ }^{\mathrm{c} *}$ Julia Ming ${ }^{\mathrm{d}}$ and \\ Guan-Fang $\mathrm{Su}^{\mathrm{a}}$
}

\begin{abstract}
a'Department of Ophthalmology, The Second Hospital of Jilin University, 218 Ziqiang Street, Changchun 130041, People's Republic of China, 'Department of Orthopedics, The China-Japan Union Hospital of Jilin University Changchun, Changchun 130033, People's Republic of China, 'Department of Vascular Surgery, The ChinaJapan Union Hospital of Jilin University, Changchun 130033, People's Republic of China, and 'St Erik's Eye Hospital, Karolinska Institutet, Polhemsgatan 50, SE-112 82 Stockholm, Sweden. *Correspondence e-mail: drsundj@163.com
\end{abstract}

Received 27 September 2014; accepted 2 October 2014

Edited by E. R. T. Tiekink, University of Malaya, Malaysia

In the title salt, $\left[\mathrm{Zn}\left(\mathrm{C}_{6} \mathrm{H}_{15} \mathrm{NO}_{3}\right)_{2}\right]\left(\mathrm{C}_{8} \mathrm{H}_{4} \mathrm{O}_{4}\right)$, the $\mathrm{Zn}^{\mathrm{II}}$ cation, located on a centre of inversion, is coordinated by four $\mathrm{O}$ atoms and two $\mathrm{N}$ atoms from two tridentate 2-[bis(2hydroxyethyl)amino]ethanol (BHEA) ligands, giving rise to a slightly distorted octahedral geometry. The terephthalate dianion, located about a centre of inversion, is not coordinated to $\mathrm{Zn}^{\mathrm{II}}$ but is connected through $\mathrm{O}-\mathrm{H} \cdots \mathrm{O}$ contacts with $\left[\mathrm{Zn}(\mathrm{BHEA})_{2}\right]^{2+}$ cations, leading to a three-dimensional crystal structure.

Keywords: crystal structure; chelate; hydrogen bonding; terephthalate.

CCDC reference: 1027329

\section{Related literature}

For background and a related structure, see: Hamamci et al. (2002).

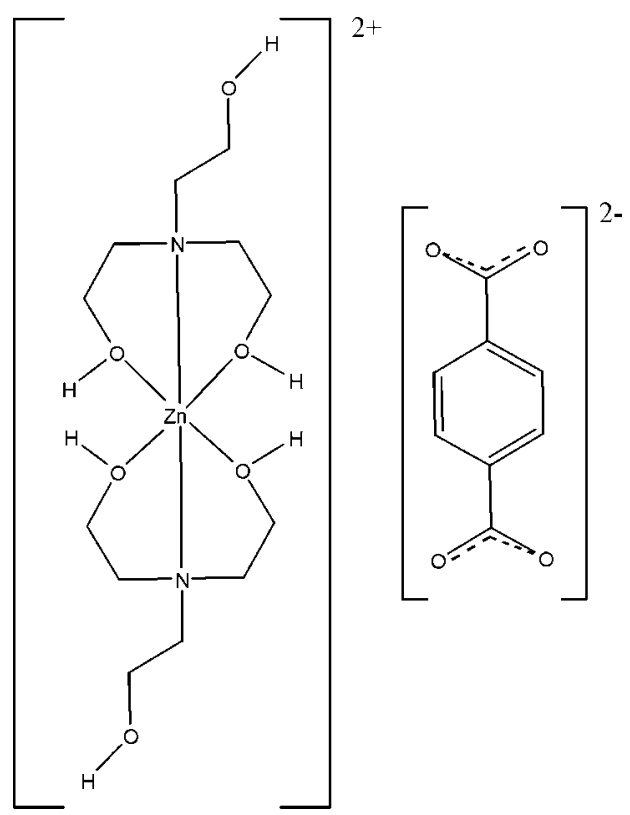

\section{Experimental}

\subsection{Crystal data}

$\left[\mathrm{Zn}\left(\mathrm{C}_{6} \mathrm{H}_{15} \mathrm{NO}_{3}\right)_{2}\right]\left(\mathrm{C}_{8} \mathrm{H}_{4} \mathrm{O}_{4}\right)$

$M_{r}=527.86$

Triclinic, $P \overline{1}$

$a=7.963$ (5) ̊

$b=8.823(5) \AA$

$c=9.198(5) \AA$

$\alpha=89.315(5)^{\circ}$

$\beta=72.421(5)^{\circ}$

$\gamma=66.208(5)^{\circ}$

$V=559.2(6) \AA^{3}$

$Z=1$

Mo $K \alpha$ radiation

$\mu=1.16 \mathrm{~mm}^{-1}$

$T=293 \mathrm{~K}$

$0.26 \times 0.24 \times 0.23 \mathrm{~mm}$

\subsection{Data collection}

Bruker SMART APEXII CCD diffractometer

Absorption correction: multi-scan (SADABS; Bruker, 2002)

$T_{\min }=0.725, T_{\max }=0.803$

3145 measured reflections 2189 independent reflections 2165 reflections with $I>2 \sigma(I)$ $R_{\text {int }}=0.012$

\subsection{Refinement}

$R\left[F^{2}>2 \sigma\left(F^{2}\right)\right]=0.027$

$w R\left(F^{2}\right)=0.072$

$S=1.10$

2189 reflections

160 parameters

3 restraints

$\mathrm{H}$ atoms treated by a mixture of independent and constrained refinement

$\Delta \rho_{\max }=0.45 \mathrm{e}^{-3}$

$\Delta \rho_{\min }=-0.51 \mathrm{e}^{-3}$

Table 1

Hydrogen-bond geometry $\left(\AA{ }^{\circ}\right)$.

\begin{tabular}{lllll}
\hline$D-\mathrm{H} \cdots A$ & $D-\mathrm{H}$ & $\mathrm{H} \cdots A$ & $D \cdots A$ & $D-\mathrm{H} \cdots A$ \\
\hline $\mathrm{O} 1-\mathrm{H} 1 \cdots \mathrm{O}^{\mathrm{i}}$ & $0.82(2)$ & $1.82(2)$ & $2.632(2)$ & $177(2)$ \\
$\mathrm{O} 2-\mathrm{H} 2 \cdots 4^{\mathrm{ii}}$ & $0.83(2)$ & $1.74(2)$ & $2.564(2)$ & $178(2)$ \\
$\mathrm{O} 3-\mathrm{H} 3 \cdots$ O5 $^{\text {iii }}$ & $0.87(2)$ & $2.13(2)$ & $2.942(3)$ & $155(2)$ \\
\hline Symmetry codes: & (i) $-x+2,-y-1,-z ;$ & (ii) $x-1, y+1, z ; \quad$ (iii) \\
$-x+2,-y-1,-z+1$. & & &
\end{tabular}




\section{data reports}

Data collection: APEX2 (Bruker, 2002); cell refinement: SAINT (Bruker, 2002); data reduction: $S A I N T$; $\operatorname{program}(\mathrm{s})$ used to solve structure: SHELXS97 (Sheldrick, 2008); program(s) used to refine structure: SHELXL97 (Sheldrick, 2008); molecular graphics: SHELXTL (Sheldrick, 2008); software used to prepare material for publication: SHELXTL and publCIF (Westrip, 2010).

\section{Acknowledgements}

The project is supported by the International Scientific and Technological Cooperation Foundation of Jilin Province (20120722).
Supporting information for this paper is available from the $\mathrm{IUCr}$ electronic archives (Reference: TK5343).

\section{References}

Bruker (2002). APEX2, SAINT and SADABS. Bruker AXS Inc., Madison, Wisconsin, USA.

Hamamci, S., Yilmaz, V. T. \& Thöne, C. (2002). Acta Cryst. E58, m369-m371. Sheldrick, G. M. (2008). Acta Cryst. A64, 112-122.

Westrip, S. P. (2010). J. Appl. Cryst. 43, 920-925. 


\section{supporting information}

Acta Cryst. (2014). E70, m361-m362 [doi:10.1107/S1600536814021771]

\section{Crystal structure of bis $\left\{2-[b i s(2-h y d r o x y e t h y l) a m i n o]\right.$ ethanol- $\left.\kappa^{3} O, N, O^{\prime}\right\}$ zinc terephthalate}

\section{Ya-Ping Li, Hu Zang, Dajun Sun, Julia Ming and Guan-Fang Su}

\section{S1. Preparation}

The synthesis was performed under hydrothermal conditions. A mixture of $\mathrm{Zn}\left(\mathrm{CH}_{3} \mathrm{COO}\right)_{2} 2\left(\mathrm{H}_{2} \mathrm{O}\right),(0.2 \mathrm{mmol}, 0.044 \mathrm{~g})$, 2-[bis(2-hydroxyethyl)amino]ethanol $(0.4 \mathrm{mmol}, 0.062 \mathrm{~g})$, sodium terephthalate $(0.2 \mathrm{mmol}, 0.042 \mathrm{~g})$ and $\mathrm{H}_{2} \mathrm{O}(20 \mathrm{~mL})$ in a $30 \mathrm{~mL}$ stainless steel reactor with a Teflon liner was heated from 293 to $433 \mathrm{~K}$ in $2 \mathrm{~h}$ and then held at a constant temperature of $433 \mathrm{~K}$ for $72 \mathrm{~h}$, after which the mixture was cooled to $298 \mathrm{~K}$. Colourless crystals of the title compound were recovered from the reaction.

\section{S2. Refinement}

All C-bound $\mathrm{H}$ atoms were positioned with idealized geometry $(0.93-0.97 \AA)$ and refined isotropically with $U_{\text {iso }}(\mathrm{H})=1.2$ $U_{\text {eq }}(\mathrm{C})$ using a riding model. The hydroxy $\mathrm{H}$-atoms were located in a different Fourier map and were refined with an $\mathrm{O}-$ $\mathrm{H}$ distance restrained to 0.85 (2) $\AA$ and with $U_{\text {iso }}(\mathrm{H})=1.5 U_{\text {eq }}(\mathrm{O})$.

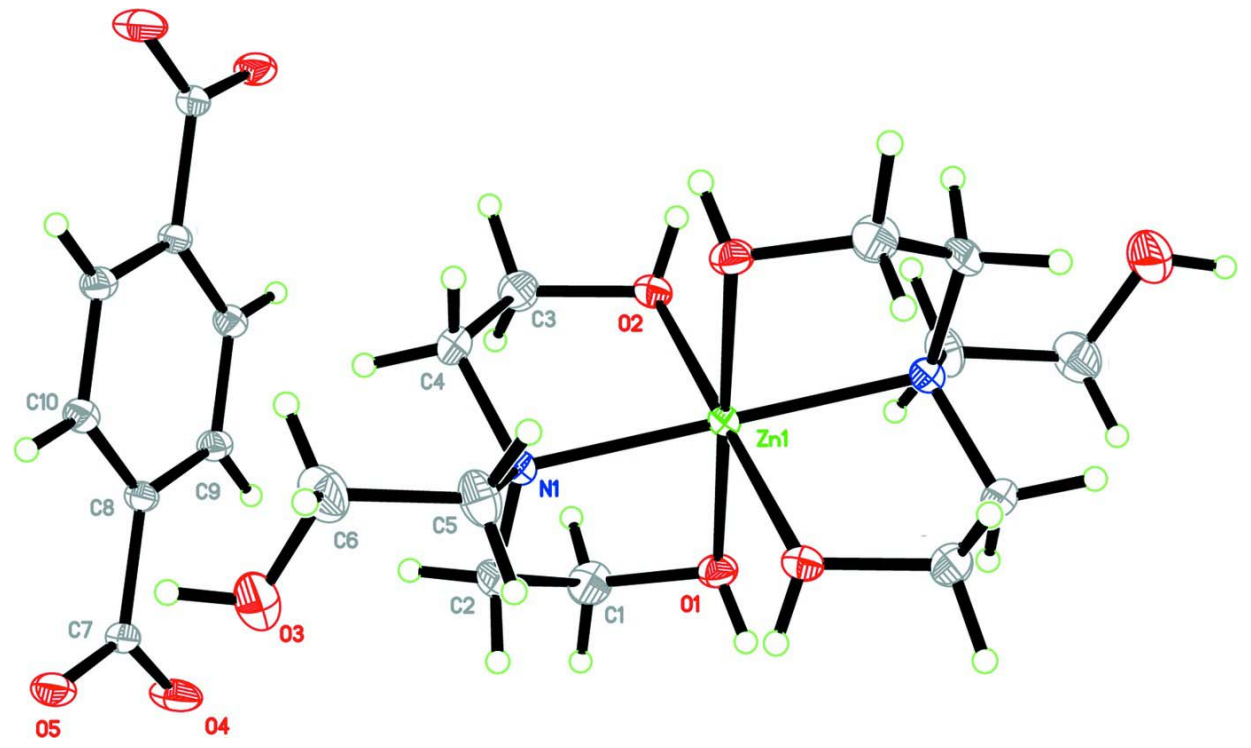

Figure 1

A view of the ions in the title salt. Displacement ellipsoids are drawn at the $30 \%$ probability level. Unlabelled atoms in the cation are related by $1-x,-y,-z$, and those in the dianion by $1-x,-1-y, 1-z$. 


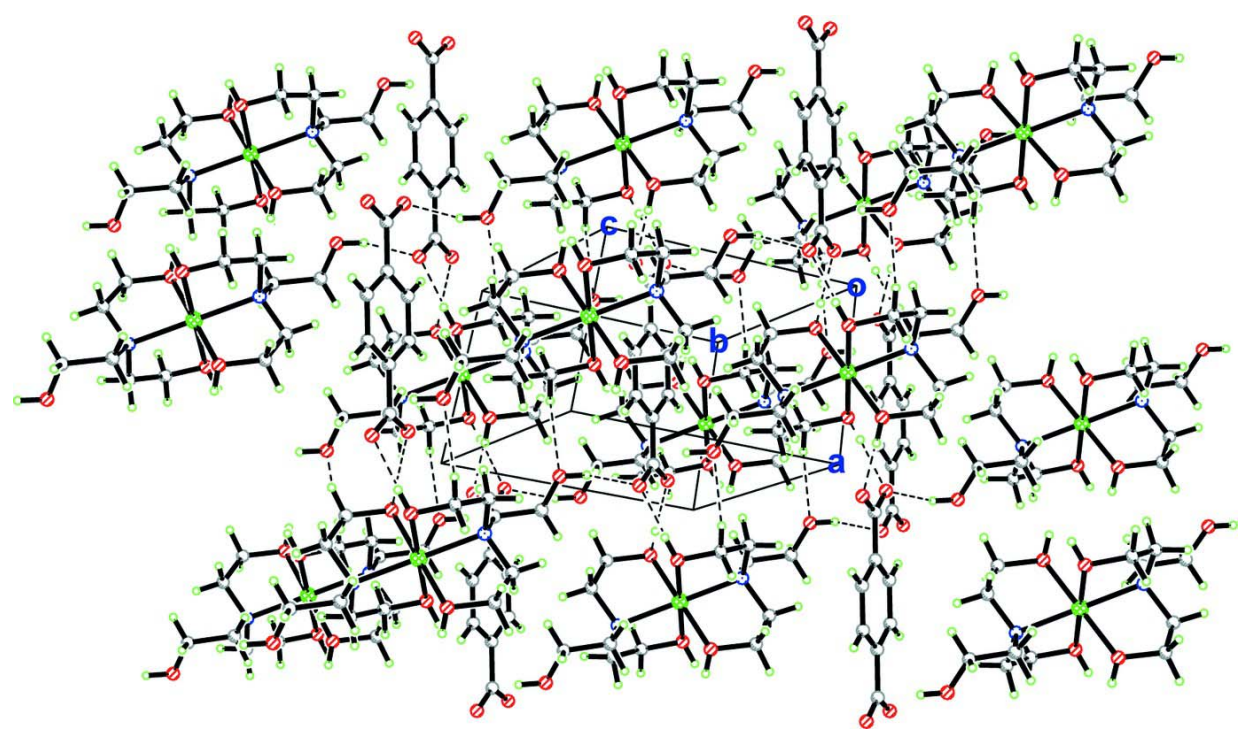

Figure 2

A view of the crystal structure of the title salt. Hydrogen bonds are shown as dashed lines.

\section{Bis\{2-[bis(2-hydroxyethyl)amino] ethanol- $\left.\kappa^{3} O, N, O^{\prime}\right\}$ zinc terephthalate}

\section{Crystal data}

$\left[\mathrm{Zn}\left(\mathrm{C}_{6} \mathrm{H}_{15} \mathrm{NO}_{3}\right)_{2}\right]\left(\mathrm{C}_{8} \mathrm{H}_{4} \mathrm{O}_{4}\right)$

$M_{r}=527.86$

Triclinic, $P \overline{1}$

Hall symbol: -P 1

$a=7.963(5) \AA$

$b=8.823(5) \AA$

$c=9.198(5) \AA$

$\alpha=89.315(5)^{\circ}$

$\beta=72.421(5)^{\circ}$

$\gamma=66.208(5)^{\circ}$

$V=559.2(6) \AA^{3}$

Data collection

Bruker SMART APEXII CCD

diffractometer

Radiation source: fine-focus sealed tube

Graphite monochromator

$\varphi$ and $\omega$ scans

Absorption correction: multi-scan

(SADABS; Bruker, 2002)

$T_{\min }=0.725, T_{\max }=0.803$

Refinement

Refinement on $F^{2}$

Least-squares matrix: full

$R\left[F^{2}>2 \sigma\left(F^{2}\right)\right]=0.027$

$w R\left(F^{2}\right)=0.072$

$S=1.10$

2189 reflections

160 parameters
$Z=1$

$F(000)=278$

$D_{\mathrm{x}}=1.567 \mathrm{Mg} \mathrm{m}^{-3}$

Mo $K \alpha$ radiation, $\lambda=0.71069 \AA$

Cell parameters from 2165 reflections

$\theta=1.7-22.8^{\circ}$

$\mu=1.16 \mathrm{~mm}^{-1}$

$T=293 \mathrm{~K}$

Block, colourless

$0.26 \times 0.24 \times 0.23 \mathrm{~mm}$

3145 measured reflections

2189 independent reflections

2165 reflections with $I>2 \sigma(I)$

$R_{\text {int }}=0.012$

$\theta_{\text {max }}=26.2^{\circ}, \theta_{\min }=2.3^{\circ}$

$h=-9 \rightarrow 8$

$k=-10 \rightarrow 10$

$l=-10 \rightarrow 11$

3 restraints

Primary atom site location: structure-invariant direct methods

Secondary atom site location: difference Fourier map

Hydrogen site location: inferred from neighbouring sites 
$\mathrm{H}$ atoms treated by a mixture of independent and constrained refinement

$w=1 /\left[\sigma^{2}\left(F_{\mathrm{o}}^{2}\right)+(0.0381 P)^{2}+0.3177 P\right]$

where $P=\left(F_{\mathrm{o}}^{2}+2 F_{\mathrm{c}}^{2}\right) / 3$

$$
\begin{aligned}
& (\Delta / \sigma)_{\max }<0.001 \\
& \Delta \rho_{\max }=0.45 \mathrm{e}^{-3} \\
& \Delta \rho_{\min }=-0.51 \mathrm{e} \AA^{-3}
\end{aligned}
$$

\section{Special details}

Geometry. All esds (except the esd in the dihedral angle between two 1.s. planes) are estimated using the full covariance matrix. The cell esds are taken into account individually in the estimation of esds in distances, angles and torsion angles; correlations between esds in cell parameters are only used when they are defined by crystal symmetry. An approximate (isotropic) treatment of cell esds is used for estimating esds involving l.s. planes.

Refinement. Refinement of $\mathrm{F}^{2}$ against ALL reflections. The weighted R-factor $\mathrm{wR}$ and goodness of fit $\mathrm{S}$ are based on $\mathrm{F}^{2}$, conventional R-factors $R$ are based on $F$, with $F$ set to zero for negative $F^{2}$. The threshold expression of $F^{2}>2$ sigma $\left(F^{2}\right)$ is used only for calculating R-factors ( $\mathrm{gt}$ ) etc. and is not relevant to the choice of reflections for refinement. R-factors based

\begin{tabular}{|c|c|c|c|c|}
\hline & $x$ & $y$ & $z$ & $U_{\text {iso }} * / U_{\text {eq }}$ \\
\hline $\mathrm{C} 1$ & 0.6774 & $-0.3639(2)$ & $-0.0601(2)$ & $0.0370(5)$ \\
\hline H1A & 0.7747 & -0.4664 & -0.1248 & $0.044 *$ \\
\hline H1B & 0.5597 & -0.3796 & -0.0138 & $0.044^{*}$ \\
\hline $\mathrm{C} 2$ & $0.7484(3)$ & $-0.3279(2)$ & $0.0655(2)$ & $0.0331(4)$ \\
\hline $\mathrm{H} 2 \mathrm{~A}$ & 0.7475 & -0.4087 & 0.1378 & $0.040 *$ \\
\hline $\mathrm{H} 2 \mathrm{~B}$ & 0.8814 & -0.3417 & 0.0199 & $0.040 *$ \\
\hline $\mathrm{C} 3$ & 0.3010 & -0.1603 & $0.2345(2)$ & $0.0363(5)$ \\
\hline H3A & 0.3374 & -0.2759 & 0.1998 & $0.044 *$ \\
\hline H3B & 0.1832 & -0.1228 & 0.3224 & $0.044 *$ \\
\hline $\mathrm{C} 4$ & $0.4611(3)$ & -0.1470 & $0.2818(2)$ & $0.0325(4)$ \\
\hline H4A & 0.4091 & -0.0417 & 0.3462 & $0.039 *$ \\
\hline H4B & 0.5057 & -0.2361 & 0.3426 & $0.039^{*}$ \\
\hline $\mathrm{C} 5$ & 0.7460 & $-0.0863(3)$ & 0.2013 & $0.0371(5)$ \\
\hline H5A & 0.6741 & 0.0341 & 0.2208 & $0.044^{*}$ \\
\hline H5B & 0.8648 & -0.1107 & 0.1168 & $0.044 *$ \\
\hline C6 & $0.8006(4)$ & $-0.1462(3)$ & 0.3430 & $0.0463(5)$ \\
\hline H6A & 0.8579 & -0.0793 & 0.3745 & $0.056^{*}$ \\
\hline H6B & 0.6851 & -0.1329 & 0.4268 & $0.056^{*}$ \\
\hline $\mathrm{C} 7$ & $0.9062(2)$ & $-0.7092(2)$ & $0.3337(2)$ & $0.0260(4)$ \\
\hline $\mathrm{C} 8$ & $0.6957(2)$ & $-0.5992(2)$ & $0.4202(2)$ & 0.0228 \\
\hline C9 & $0.5509(3)$ & $-0.6072(2)$ & $0.3698(2)$ & $0.0253(4)$ \\
\hline H9 & 0.5846 & -0.6789 & 0.2823 & $0.030 *$ \\
\hline $\mathrm{C} 10$ & $0.6436(3)$ & -0.4910 & $0.5508(2)$ & $0.0250(4)$ \\
\hline $\mathrm{H} 10$ & 0.7392 & -0.4844 & 0.5852 & $0.030 *$ \\
\hline N1 & $0.6286(2)$ & $-0.15730(18)$ & $0.14967(17)$ & $0.0229(3)$ \\
\hline $\mathrm{O} 1$ & 0.63918 (19) & $-0.23022(16)$ & $-0.15252(15)$ & $0.0273(3)$ \\
\hline $\mathrm{O} 2$ & $0.26592(18)$ & $-0.06108(17)$ & $0.11409(15)$ & 0.0283 \\
\hline $\mathrm{O} 3$ & $0.9319(3)$ & $-0.3128(2)$ & $0.3114(2)$ & $0.0553(5)$ \\
\hline $\mathrm{O} 4$ & $0.9393(2)$ & $-0.8061(2)$ & $0.21957(18)$ & $0.0411(4)$ \\
\hline $\mathrm{O} 5$ & $1.03466(19)$ & $-0.69733(19)$ & $0.37935(16)$ & $0.0357(3)$ \\
\hline $\mathrm{Zn} 1$ & 0.5000 & 0.0000 & 0.0000 & $0.01988(10)$ \\
\hline
\end{tabular}
on $\mathrm{F}^{2}$ are statistically about twice as large as those based on $\mathrm{F}$, and R-factors based on ALL data will be even larger.

Fractional atomic coordinates and isotropic or equivalent isotropic displacement parameters $\left(\AA^{2}\right)$ 
supporting information

\begin{tabular}{lllll}
$\mathrm{H} 1$ & $0.738(3)$ & $-0.249(3)$ & $-0.224(2)$ & $0.030^{*}$ \\
$\mathrm{H} 2$ & $0.160(3)$ & $0.020(2)$ & $0.150(3)$ & $0.030^{*}$ \\
$\mathrm{H} 3$ & $0.954(3)$ & $-0.343(3)$ & $0.397(2)$ & $0.030^{*}$ \\
\hline
\end{tabular}

Atomic displacement parameters $\left(\AA^{2}\right)$

\begin{tabular}{lllllll}
\hline & $U^{11}$ & $U^{22}$ & $U^{33}$ & $U^{12}$ & $U^{13}$ & $U^{23}$ \\
\hline C1 & $0.0491(12)$ & $0.0214(9)$ & $0.0346(10)$ & $-0.0125(9)$ & $-0.0086(9)$ & $-0.0021(8)$ \\
C2 & $0.0334(10)$ & $0.0202(9)$ & $0.0327(10)$ & $-0.0003(7)$ & $-0.0084(8)$ & $0.0034(7)$ \\
C3 & $0.0277(9)$ & $0.0398(11)$ & $0.0383(11)$ & $-0.0150(9)$ & $-0.0056(8)$ & $0.0149(9)$ \\
C4 & $0.0298(9)$ & $0.0384(10)$ & $0.0218(9)$ & $-0.0095(8)$ & $-0.0049(7)$ & $0.0076(8)$ \\
C5 & $0.0427(11)$ & $0.0357(11)$ & $0.0466(12)$ & $-0.0202(9)$ & $-0.0284(10)$ & $0.0128(9)$ \\
C6 & $0.0430(12)$ & $0.0505(14)$ & $0.0519(14)$ & $-0.0171(11)$ & $-0.0281(11)$ & $0.0066(11)$ \\
C7 & $0.0202(8)$ & $0.0259(9)$ & $0.0237(8)$ & $-0.0041(7)$ & $-0.0038(7)$ & $0.0040(7)$ \\
C8 & $0.0184(8)$ & $0.0208(8)$ & $0.0238(8)$ & $-0.0053(6)$ & $-0.0038(6)$ & $0.0041(6)$ \\
C9 & $0.0231(8)$ & $0.0249(9)$ & $0.0233(8)$ & $-0.0071(7)$ & $-0.0055(7)$ & $-0.0011(6)$ \\
C10 & $0.0205(8)$ & $0.0271(9)$ & $0.0263(9)$ & $-0.0084(7)$ & $-0.0082(7)$ & $0.0027(7)$ \\
N1 & $0.0215(7)$ & $0.0215(7)$ & $0.0236(7)$ & $-0.0066(6)$ & $-0.0078(6)$ & $0.0031(6)$ \\
O1 & $0.0249(6)$ & $0.0268(6)$ & $0.0237(6)$ & $-0.0075(5)$ & $-0.0038(5)$ & $-0.0028(5)$ \\
O2 & $0.0189(6)$ & $0.0293(7)$ & $0.0303(7)$ & $-0.0059(5)$ & $-0.0050(5)$ & $0.0047(5)$ \\
O3 & $0.0529(10)$ & $0.0576(11)$ & $0.0483(10)$ & $-0.0096(9)$ & $-0.0261(9)$ & $0.0130(8)$ \\
O4 & $0.0226(7)$ & $0.0423(8)$ & $0.0432(8)$ & $-0.0025(6)$ & $-0.0049(6)$ & $-0.0157(7)$ \\
O5 & $0.0188(6)$ & $0.0481(9)$ & $0.0311(7)$ & $-0.0068(6)$ & $-0.0056(5)$ & $-0.0038(6)$ \\
Zn1 & $0.01853(15)$ & $0.01801(15)$ & $0.01999(15)$ & $-0.00478(11)$ & $-0.00604(10)$ & $0.00264(10)$ \\
& & & & & & \\
\hline
\end{tabular}

Geometric parameters $\left(A,{ }^{\circ}\right)$

\begin{tabular}{llll}
\hline $\mathrm{C} 1-\mathrm{O} 1$ & $1.431(2)$ & $\mathrm{C} 6-\mathrm{H} 6 \mathrm{~B}$ & 0.9700 \\
$\mathrm{C} 1-\mathrm{C} 2$ & $1.518(3)$ & $\mathrm{C} 7-\mathrm{O} 4$ & $1.255(2)$ \\
$\mathrm{C} 1-\mathrm{H} 1 \mathrm{~A}$ & 0.9700 & $\mathrm{C} 7-\mathrm{O} 5$ & $1.257(2)$ \\
$\mathrm{C} 1-\mathrm{H} 1 \mathrm{~B}$ & 0.9700 & $\mathrm{C} 7-\mathrm{C} 8$ & $1.511(2)$ \\
$\mathrm{C} 2-\mathrm{N} 1$ & $1.483(2)$ & $\mathrm{C} 8-\mathrm{C} 10$ & $1.390(3)$ \\
$\mathrm{C} 2-\mathrm{H} 2 \mathrm{~A}$ & 0.9700 & $\mathrm{C} 8-\mathrm{C} 9$ & $1.393(3)$ \\
$\mathrm{C} 2-\mathrm{H} 2 \mathrm{~B}$ & 0.9700 & $\mathrm{C} 9-\mathrm{C} 10^{\mathrm{i}}$ & $1.390(3)$ \\
$\mathrm{C} 3-\mathrm{O} 2$ & $1.427(2)$ & $\mathrm{C} 9-\mathrm{H} 9$ & 0.9300 \\
$\mathrm{C} 3-\mathrm{C} 4$ & $1.511(3)$ & $\mathrm{C} 10-\mathrm{C} 9^{\mathrm{i}}$ & $1.390(3)$ \\
$\mathrm{C} 3-\mathrm{H} 3 \mathrm{~A}$ & 0.9700 & $\mathrm{C} 10-\mathrm{H} 10$ & 0.9300 \\
$\mathrm{C} 3-\mathrm{H} 3 \mathrm{~B}$ & 0.9700 & $\mathrm{~N} 1-\mathrm{Zn} 1$ & $2.1282(16)$ \\
$\mathrm{C} 4-\mathrm{N} 1$ & $1.480(2)$ & $\mathrm{O} 1-\mathrm{Zn} 1$ & $2.1529(16)$ \\
$\mathrm{C} 4-\mathrm{H} 4 \mathrm{~A}$ & 0.9700 & $\mathrm{O} 1-\mathrm{H} 1$ & $0.815(16)$ \\
$\mathrm{C} 4-\mathrm{H} 4 \mathrm{~B}$ & 0.9700 & $\mathrm{O} 2-\mathrm{Zn} 1$ & $2.1169(16)$ \\
$\mathrm{C} 5-\mathrm{N} 1$ & $1.496(2)$ & $\mathrm{O} 2-\mathrm{H} 2$ & $0.825(16)$ \\
$\mathrm{C} 5-\mathrm{C} 6$ & $1.519(3)$ & $\mathrm{O} 3-\mathrm{H} 3$ & $2.870(16)$ \\
$\mathrm{C} 5-\mathrm{H} 5 \mathrm{~A}$ & 0.9700 & $\mathrm{Zn} 1-\mathrm{O} 2^{\mathrm{ii}}$ & $2.1282(16)$ \\
$\mathrm{C} 5-\mathrm{H} 5 \mathrm{~B}$ & 0.9700 & $\mathrm{Zn} 1-\mathrm{N} 1^{\mathrm{ii}}$ & $2.1529(16)$ \\
$\mathrm{C} 6-\mathrm{O} 3$ & $1.388(3)$ & $\mathrm{Zn} 1-\mathrm{O} 1^{\mathrm{ii}}$ & \\
C6-H6A & 0.9700 & &
\end{tabular}




\begin{tabular}{|c|c|c|c|}
\hline $\mathrm{O} 1-\mathrm{C} 1-\mathrm{C} 2$ & $111.27(16)$ & $\mathrm{O} 5-\mathrm{C} 7-\mathrm{C} 8$ & $118.64(16)$ \\
\hline $\mathrm{O} 1-\mathrm{C} 1-\mathrm{H} 1 \mathrm{~A}$ & 109.4 & $\mathrm{C} 10-\mathrm{C} 8-\mathrm{C} 9$ & $119.17(16)$ \\
\hline $\mathrm{C} 2-\mathrm{C} 1-\mathrm{H} 1 \mathrm{~A}$ & 109.4 & $\mathrm{C} 10-\mathrm{C} 8-\mathrm{C} 7$ & $121.09(16)$ \\
\hline $\mathrm{O} 1-\mathrm{C} 1-\mathrm{H} 1 \mathrm{~B}$ & 109.4 & $\mathrm{C} 9-\mathrm{C} 8-\mathrm{C} 7$ & $119.74(16)$ \\
\hline $\mathrm{C} 2-\mathrm{C} 1-\mathrm{H} 1 \mathrm{~B}$ & 109.4 & $\mathrm{C} 10^{\mathrm{i}}-\mathrm{C} 9-\mathrm{C} 8$ & $120.58(17)$ \\
\hline $\mathrm{H} 1 \mathrm{~A}-\mathrm{C} 1-\mathrm{H} 1 \mathrm{~B}$ & 108.0 & $\mathrm{C} 10^{\mathrm{i}}-\mathrm{C} 9-\mathrm{H} 9$ & 119.7 \\
\hline $\mathrm{N} 1-\mathrm{C} 2-\mathrm{C} 1$ & $112.93(16)$ & $\mathrm{C} 8-\mathrm{C} 9-\mathrm{H} 9$ & 119.7 \\
\hline $\mathrm{N} 1-\mathrm{C} 2-\mathrm{H} 2 \mathrm{~A}$ & 109.0 & $\mathrm{C} 99^{\mathrm{i}}-\mathrm{C} 10-\mathrm{C} 8$ & $120.26(17)$ \\
\hline $\mathrm{C} 1-\mathrm{C} 2-\mathrm{H} 2 \mathrm{~A}$ & 109.0 & $\mathrm{C} 9-\mathrm{C} 10-\mathrm{H} 10$ & 119.9 \\
\hline $\mathrm{N} 1-\mathrm{C} 2-\mathrm{H} 2 \mathrm{~B}$ & 109.0 & $\mathrm{C} 8-\mathrm{C} 10-\mathrm{H} 10$ & 119.9 \\
\hline $\mathrm{C} 1-\mathrm{C} 2-\mathrm{H} 2 \mathrm{~B}$ & 109.0 & $\mathrm{C} 4-\mathrm{N} 1-\mathrm{C} 2$ & $113.25(15)$ \\
\hline $\mathrm{H} 2 \mathrm{~A}-\mathrm{C} 2-\mathrm{H} 2 \mathrm{~B}$ & 107.8 & $\mathrm{C} 4-\mathrm{N} 1-\mathrm{C} 5$ & $109.91(16)$ \\
\hline $\mathrm{O} 2-\mathrm{C} 3-\mathrm{C} 4$ & $110.59(16)$ & $\mathrm{C} 2-\mathrm{N} 1-\mathrm{C} 5$ & $112.28(16)$ \\
\hline $\mathrm{O} 2-\mathrm{C} 3-\mathrm{H} 3 \mathrm{~A}$ & 109.5 & $\mathrm{C} 4-\mathrm{N} 1-\mathrm{Zn} 1$ & $104.11(11)$ \\
\hline $\mathrm{C} 4-\mathrm{C} 3-\mathrm{H} 3 \mathrm{~A}$ & 109.5 & $\mathrm{C} 2-\mathrm{N} 1-\mathrm{Zn} 1$ & $108.27(12)$ \\
\hline $\mathrm{O} 2-\mathrm{C} 3-\mathrm{H} 3 \mathrm{~B}$ & 109.5 & $\mathrm{C} 5-\mathrm{N} 1-\mathrm{Zn} 1$ & $108.59(12)$ \\
\hline $\mathrm{C} 4-\mathrm{C} 3-\mathrm{H} 3 \mathrm{~B}$ & 109.5 & $\mathrm{C} 1-\mathrm{O} 1-\mathrm{Zn} 1$ & $107.34(11)$ \\
\hline $\mathrm{H} 3 \mathrm{~A}-\mathrm{C} 3-\mathrm{H} 3 \mathrm{~B}$ & 108.1 & $\mathrm{C} 1-\mathrm{O} 1-\mathrm{H} 1$ & $108.2(17)$ \\
\hline $\mathrm{N} 1-\mathrm{C} 4-\mathrm{C} 3$ & $113.26(16)$ & $\mathrm{Zn} 1-\mathrm{O} 1-\mathrm{H} 1$ & $120.1(16)$ \\
\hline $\mathrm{N} 1-\mathrm{C} 4-\mathrm{H} 4 \mathrm{~A}$ & 108.9 & $\mathrm{C} 3-\mathrm{O} 2-\mathrm{Zn} 1$ & $112.52(11)$ \\
\hline $\mathrm{C} 3-\mathrm{C} 4-\mathrm{H} 4 \mathrm{~A}$ & 108.9 & $\mathrm{C} 3-\mathrm{O} 2-\mathrm{H} 2$ & $108.0(16)$ \\
\hline $\mathrm{N} 1-\mathrm{C} 4-\mathrm{H} 4 \mathrm{~B}$ & 108.9 & $\mathrm{Zn} 1-\mathrm{O} 2-\mathrm{H} 2$ & $114.6(16)$ \\
\hline $\mathrm{C} 3-\mathrm{C} 4-\mathrm{H} 4 \mathrm{~B}$ & 108.9 & $\mathrm{C} 6-\mathrm{O} 3-\mathrm{H} 3$ & $106.6(15)$ \\
\hline $\mathrm{H} 4 \mathrm{~A}-\mathrm{C} 4-\mathrm{H} 4 \mathrm{~B}$ & 107.7 & $\mathrm{O} 2^{\mathrm{ii}}-\mathrm{Zn} 1-\mathrm{O} 2$ & $180.00(4)$ \\
\hline $\mathrm{N} 1-\mathrm{C} 5-\mathrm{C} 6$ & $117.31(18)$ & 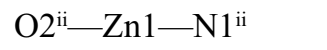 & $81.99(7)$ \\
\hline $\mathrm{N} 1-\mathrm{C} 5-\mathrm{H} 5 \mathrm{~A}$ & 108.0 & $\mathrm{O} 2-\mathrm{Zn} 1-\mathrm{N} 1^{\mathrm{ii}}$ & $98.01(7)$ \\
\hline $\mathrm{C} 6-\mathrm{C} 5-\mathrm{H} 5 \mathrm{~A}$ & 108.0 & 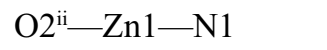 & $98.01(7)$ \\
\hline $\mathrm{N} 1-\mathrm{C} 5-\mathrm{H} 5 \mathrm{~B}$ & 108.0 & $\mathrm{O} 2-\mathrm{Zn} 1-\mathrm{N} 1$ & $81.99(7)$ \\
\hline $\mathrm{C} 6-\mathrm{C} 5-\mathrm{H} 5 \mathrm{~B}$ & 108.0 & $\mathrm{~N} 1{ }^{\mathrm{ii}}-\mathrm{Zn} 1-\mathrm{N} 1$ & 180.0 \\
\hline $\mathrm{H} 5 \mathrm{~A}-\mathrm{C} 5-\mathrm{H} 5 \mathrm{~B}$ & 107.2 & $\mathrm{O} 2^{\mathrm{ii}}-\mathrm{Zn} 1-\mathrm{O} 1^{\mathrm{ii}}$ & $90.41(6)$ \\
\hline $\mathrm{O} 3-\mathrm{C} 6-\mathrm{C} 5$ & $110.1(2)$ & $\mathrm{O} 2-\mathrm{Zn} 1-\mathrm{O} 1^{\mathrm{ii}}$ & $89.59(6)$ \\
\hline $\mathrm{O} 3-\mathrm{C} 6-\mathrm{H} 6 \mathrm{~A}$ & 109.6 & 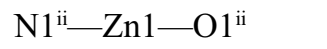 & $82.73(7)$ \\
\hline $\mathrm{C} 5-\mathrm{C} 6-\mathrm{H} 6 \mathrm{~A}$ & 109.6 & $\mathrm{~N} 1-\mathrm{Zn} 1-\mathrm{O} 1^{\mathrm{ii}}$ & $97.27(7)$ \\
\hline $\mathrm{O} 3-\mathrm{C} 6-\mathrm{H} 6 \mathrm{~B}$ & 109.6 & $\mathrm{O} 2^{\mathrm{ii}}-\mathrm{Zn} 1-\mathrm{O} 1$ & $89.59(6)$ \\
\hline $\mathrm{C} 5-\mathrm{C} 6-\mathrm{H} 6 \mathrm{~B}$ & 109.6 & $\mathrm{O} 2-\mathrm{Zn} 1-\mathrm{O} 1$ & $90.41(6)$ \\
\hline $\mathrm{H} 6 \mathrm{~A}-\mathrm{C} 6-\mathrm{H} 6 \mathrm{~B}$ & 108.2 & 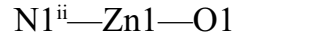 & $97.27(7)$ \\
\hline $\mathrm{O} 4-\mathrm{C} 7-\mathrm{O} 5$ & $124.72(16)$ & $\mathrm{N} 1-\mathrm{Zn} 1-\mathrm{O} 1$ & $82.73(7)$ \\
\hline $\mathrm{O} 4-\mathrm{C} 7-\mathrm{C} 8$ & $116.64(16)$ & $\mathrm{O} 1^{\mathrm{ii}}-\mathrm{Zn} 1-\mathrm{O} 1$ & $180.00(12)$ \\
\hline $\mathrm{O} 1-\mathrm{C} 1-\mathrm{C} 2-\mathrm{N} 1$ & $47.2(2)$ & $\mathrm{C} 3-\mathrm{O} 2-\mathrm{Zn} 1-\mathrm{N} 1$ & $4.13(13)$ \\
\hline $\mathrm{O} 2-\mathrm{C} 3-\mathrm{C} 4-\mathrm{N} 1$ & $-43.7(2)$ & $\mathrm{C} 3-\mathrm{O} 2-\mathrm{Zn} 1-\mathrm{O} 1^{\mathrm{ii}}$ & $-93.26(13)$ \\
\hline $\mathrm{N} 1-\mathrm{C} 5-\mathrm{C} 6-\mathrm{O} 3$ & $68.3(3)$ & $\mathrm{C} 3-\mathrm{O} 2-\mathrm{Zn} 1-\mathrm{O} 1$ & $86.74(13)$ \\
\hline $\mathrm{O} 4-\mathrm{C} 7-\mathrm{C} 8-\mathrm{C} 10$ & $178.15(17)$ & $\mathrm{C} 4-\mathrm{N} 1-\mathrm{Zn} 1-\mathrm{O} 2^{\mathrm{ii}}$ & $154.45(12)$ \\
\hline $\mathrm{O} 5-\mathrm{C} 7-\mathrm{C} 8-\mathrm{C} 10$ & $-2.2(3)$ & $\mathrm{C} 2-\mathrm{N} 1-\mathrm{Zn} 1-\mathrm{O} 2^{\mathrm{ii}}$ & $-84.78(12)$ \\
\hline $\mathrm{O} 4-\mathrm{C} 7-\mathrm{C} 8-\mathrm{C} 9$ & $-1.1(3)$ & $\mathrm{C} 5-\mathrm{N} 1-\mathrm{Zn} 1-\mathrm{O} 2^{\mathrm{ii}}$ & $37.38(13)$ \\
\hline $\mathrm{O} 5-\mathrm{C} 7-\mathrm{C} 8-\mathrm{C} 9$ & $178.52(17)$ & $\mathrm{C} 4-\mathrm{N} 1-\mathrm{Zn} 1-\mathrm{O} 2$ & $-25.55(12)$ \\
\hline $\mathrm{C} 10-\mathrm{C} 8-\mathrm{C} 9-\mathrm{C} 10^{\mathrm{i}}$ & $-0.3(3)$ & $\mathrm{C} 2-\mathrm{N} 1-\mathrm{Zn} 1-\mathrm{O} 2$ & $95.22(12)$ \\
\hline $\mathrm{C} 7-\mathrm{C} 8-\mathrm{C} 9-\mathrm{C} 10^{\mathrm{i}}$ & $179.03(16)$ & $\mathrm{C} 5-\mathrm{N} 1-\mathrm{Zn} 1-\mathrm{O} 2$ & $-142.62(13)$ \\
\hline
\end{tabular}




$\begin{array}{llll}\mathrm{C} 9-\mathrm{C} 8-\mathrm{C} 10-\mathrm{C} 9^{\mathrm{i}} & 0.3(3) & \mathrm{C} 4-\mathrm{N} 1-\mathrm{Zn} 1-\mathrm{N} 1^{\mathrm{ii}} & -80(100) \\ \mathrm{C} 7-\mathrm{C} 8-\mathrm{C} 10-\mathrm{C} 9^{\mathrm{i}} & -179.02(16) & \mathrm{C} 2-\mathrm{N} 1-\mathrm{Zn} 1-\mathrm{N} 1^{\mathrm{ii}} & 41(100) \\ \mathrm{C} 3-\mathrm{C} 4-\mathrm{N} 1-\mathrm{C} 2 & -72.9(2) & \mathrm{C} 5-\mathrm{N} 1-\mathrm{Zn} 1-\mathrm{N} 1^{\mathrm{ii}} & 163(100) \\ \mathrm{C} 3-\mathrm{C} 4-\mathrm{N} 1-\mathrm{C} 5 & 160.62(17) & \mathrm{C} 4-\mathrm{N} 1-\mathrm{Zn} 1-\mathrm{O} 1^{\mathrm{ii}} & 63.01(12) \\ \mathrm{C} 3-\mathrm{C} 4-\mathrm{N} 1-\mathrm{Zn} 1 & 44.47(18) & \mathrm{C} 2-\mathrm{N} 1-\mathrm{Zn} 1-\mathrm{O} 1^{\mathrm{ii}} & -176.22(12) \\ \mathrm{C} 1-\mathrm{C} 2-\mathrm{N} 1-\mathrm{C} 4 & 87.9(2) & \mathrm{C} 5-\mathrm{N} 1-\mathrm{Zn} 1-\mathrm{O} 1^{\mathrm{ii}} & -54.06(13) \\ \mathrm{C} 1-\mathrm{C} 2-\mathrm{N} 1-\mathrm{C} 5 & -146.89(18) & \mathrm{C} 4-\mathrm{N} 1-\mathrm{Zn} 1-\mathrm{O} 1 & -116.99(12) \\ \mathrm{C} 1-\mathrm{C} 2-\mathrm{N} 1-\mathrm{Zn} 1 & -27.01(19) & \mathrm{C} 2-\mathrm{N} 1-\mathrm{Zn} 1-\mathrm{O} 1 & 3.78(12) \\ \mathrm{C} 6-\mathrm{C} 5-\mathrm{N} 1-\mathrm{C} 4 & 48.9(2) & \mathrm{C} 5-\mathrm{N} 1-\mathrm{Zn} 1-\mathrm{O} 1 & 125.94(13) \\ \mathrm{C} 6-\mathrm{C} 5-\mathrm{N} 1-\mathrm{C} 2 & -78.1(2) & \mathrm{C} 1-\mathrm{O} 1-\mathrm{Zn} 1-\mathrm{O} 2^{\mathrm{ii}} & 118.61(13) \\ \mathrm{C} 6-\mathrm{C} 5-\mathrm{N} 1-\mathrm{Zn} 1 & 162.17(16) & \mathrm{C} 1-\mathrm{O} 1-\mathrm{Zn} 1-\mathrm{O} 2 & -61.39(13) \\ \mathrm{C} 2-\mathrm{C} 1-\mathrm{O} 1-\mathrm{Zn} 1 & -40.83(19) & \mathrm{C} 1-\mathrm{O} 1-\mathrm{Zn} 1-\mathrm{N} 1^{\mathrm{ii}} & -159.52(12) \\ \mathrm{C} 4-\mathrm{C} 3-\mathrm{O} 2-\mathrm{Zn} 1 & 18.7(2) & \mathrm{C} 1-\mathrm{O} 1-\mathrm{Zn} 1-\mathrm{N} 1 & 20.48(12) \\ \mathrm{C} 3-\mathrm{O} 2-\mathrm{Zn} 1-\mathrm{O} 2^{\mathrm{ii}} & 116(100) & \mathrm{C} 1-\mathrm{O} 1-\mathrm{Zn} 1-\mathrm{O} 1^{\mathrm{ii}} & -79(100) \\ \mathrm{C} 3-\mathrm{O} 2-\mathrm{Zn} 1-\mathrm{N} 1^{\mathrm{ii}} & -175.87(13) & & \end{array}$

Symmetry codes: (i) $-x+1,-y-1,-z+1$; (ii) $-x+1,-y,-z$.

Hydrogen-bond geometry $\left(\AA,{ }^{\circ}\right)$

\begin{tabular}{lllll}
\hline$D-\mathrm{H} \cdots A$ & $D-\mathrm{H}$ & $\mathrm{H} \cdots A$ & $D \cdots A$ & $D-\mathrm{H} \cdots A$ \\
\hline $\mathrm{O} 1-\mathrm{H} 1 \cdots \mathrm{O} 5^{\mathrm{iii}}$ & $0.82(2)$ & $1.82(2)$ & $2.632(2)$ & $177(2)$ \\
$\mathrm{O} 2-\mathrm{H} 2 \cdots 4^{\mathrm{iv}}$ & $0.83(2)$ & $1.74(2)$ & $2.564(2)$ & $178(2)$ \\
$\mathrm{O} 3-\mathrm{H} 3 \cdots 5^{\mathrm{v}}$ & $0.87(2)$ & $2.13(2)$ & $2.942(3)$ & $155(2)$ \\
\hline
\end{tabular}

Symmetry codes: (iii) $-x+2,-y-1,-z$; (iv) $x-1, y+1, z$; (v) $-x+2,-y-1,-z+1$. 